\title{
Method and Application of Endogenous Neurotransmitter Regulation Theory
}

\author{
Zuodong Sun ${ }^{1}$, Wuyi Sun $^{2}$, Aili Wang ${ }^{1}$ and Wenhua Wang ${ }^{2}$ \\ 1. Ya'ou Brain Science Institute of Heilongjiang Province, Harbin 150090, China \\ 2. Harbin Aobo Medical Apparatus Co., Ltd, Harbin 150090, China
}

\begin{abstract}
Objective: Verification and application of the theory of brain cell activation. Methods: Applying transcranial magnetoelectric encephalopathy treatment instrument to the treatment of neurodegenerative disease, including but not limited to: Parkinson's disease and Alzheimer's disease. Results: Transcranial magnetoelectric encephalopathy treatment instrument, its double-center, randomized, double-blind clinical trial has been carried out at the national clinical trial base, it proves that the instrument is safe and effective. This instrument is especially suitable for the treatment of the following diseases: (1) it is suitable for the treatment of mild to moderate Parkinson's disease and can significantly improve resting tremor, rigidity, bradykinesia in patients with Parkinson's disease and other symptoms; (2) it is suitable for the treatment of mild and moderate Alzheimer's disease and vascular dementia and can improve the mental state, cognitive behavior and self-care ability of daily life; (3) it is suitable for the treatment of mild to moderate depression and can obviously improve the main symptoms of depression, sleep disorder, anxiety and so on; (4) it is suitable for the treatment of cerebral apoplexy sequelae, cerebrovascular dementia and brain atrophy, and it can activate the brain cells in the state of inhibition and mobilize the potential energy of the brain. Conclusions: The theory of brain cell activation is applicable to encephalopathy and not limited to encephalopathy, according to this theory, the subject of "physical disease science" can be created.
\end{abstract}

Key words: The theory of brain cell activation, $\mathrm{Ca}^{2+}$ channel, transcranial magnetoelectric, Parkinson's disease, Alzheimer's disease.

\section{Background}

Brain science, being regarded as the last scientific tip of human beings, is the "Pearl" in the crown of scientific research. Brain diseases, especially the neuron degenerative diseases such as Parkinson's disease (PD) and Alzheimer's disease (AD), are the "most important" in brain science. They are recognized as a worldwide medical problem and are the goals that the academic world is dreaming of.

PD is closely related to the loss of dopaminergic neurons. There are about 4,000,000 patients with Parkinson disease around the world, more than 2 million in China [1-3]. In 1817, British doctor James Parkinson first discovered neuronal degenerative disease with tremor as the main symptom. At present,

Corresponding author: Zuodong Sun, Researcher, post-doctoral co-teacher; research fields: basic theoretical research in brain science and research and development of rehabilitation equipment for severe brain diseases. drugs can only control symptoms and do not cure the disease. Its increasingly prominent failure and adverse reactions have aroused widespread concern in the medical field $[4,5]$. The main methods for surgical treatment of Parkinson's disease are derogation, deep brain electrical stimulation and tissue cell transplantation. The destruction of the brain has not been advocated because of the irreversible damage to the brain. Deep brain electrical stimulation requires the implantation of foreign bodies in the brain, and there is a strict standard of selection for the patients. Tissue cell transplantation is still in exploration [6].

$\mathrm{AD}$ which is closely related to cholinergic neuron loss, persecutes 35.6 million people worldwide and adds 4.6 million annually, up from 10 million in China alone. In 1901, neuroscientist Alois Alzheimer, a German neuroscientist, discovered a neuronal degenerative disease with progressive dementia, from a female patient admitted to the hospital, which is 
Alzheimer's disease. Since 1984, Glenner and Wong have isolated $A \beta$-amyloid from senile plaque purification for more than 30 years now; $A D$ pathogenesis is still not clear. There is not any truly effective drug birth yet $[7,8]$. Some scientists pointed out in this connection: "The amyloid hypothesis may mislead the research directions of brain scientists all over the world" [9].

Especially in the past two years, the research and development of chemical drugs are once again in trouble. Global pharmaceutical giants Eli Lilly, Pfizer, Johnson \& Johnson and others have announced the failure of drug development. In particular, Pfizer announced in early 2018 the closure of the development of a new drug research and development laboratory for Parkinson's disease and Alzheimer's disease, dissolved the team of scientists, and dismissed about 300 researchers.

At present, the status quo of brain science at home and abroad is rather chaotic. The results are severely fragmented, especially in dealing with degenerative diseases of neurons; there is no core theoretical support. It can not form the main academic thought system and technology system, clinically lacks of effective treatment of such diseases, drugs, and the measures taken are "right medicine", the serious side effects of chemical drugs in turn make the patient's condition more complicated and difficult. There is no doubt that the pathogenesis must be explored, however, it is of no use to patients who are currently suffering from encephalopathy. The top priority is to put forward the scientific hypothesis or theory that can play a strategic leading role in the direction of international brain science research, invent or find techniques or methods that are effective in controlling or reversing the degenerative neurons that are developing. It is the correct idea of tackling the problem.

\section{Endogenous Neurotransmitter Regulation Theory}

Neuromodulation theory, the theory of endogenous neurotransmitter regulation, was named by the first author of this article as "the Theory of Brain Cell Activation", which is thought to have degenerative changes, is basal exocytosis that maintains their basal metabolism before they die; it is a reversible process with normal neurons. The hypothesis of that "activating brain cells is the key to the treatment of encephalopathy" [10] based on science and cell levels began in 1994. "The Theory of Brain Cell Activation" [9] published in the literature and molecular level is 2015.

The core of endogenous neurotransmitter regulation theory of $\mathrm{PD}, \mathrm{AD}$ and other neurodegenerative diseases is closely related to the physical gated ion channels, to solve with available physical means. Activation of neurons is the key to treatment. The voltage-gated $\mathrm{Ca}^{2+}$ channels are the best targets for physical activation. The purpose is to induce $\mathrm{Ca}^{2+}$ influx trigger neuronal axon terminals synaptic vesicles to release neurotransmitters [9].

Endogenous neurotransmitter regulation theory has made it clear that calcium channel is the best target of physical activation, pointed out the principles, methods and purposes of $\mathrm{PD}, \mathrm{AD}$ and other neurodegenerative diseases treatment. It clarified that the drug can not fundamentally treat the mechanism of neurodegenerative diseases, and changed people's treatment of encephalopathy that is mainly dependent on the concept of drugs and surgery. It provides the academic direction for the domestic and foreign scholars' brain science research.

Endogenous neurotransmitter regulation theory supports the vesicle hypothesis [11] and the cholinergic hypothesis [11], questions the amyloid hypothesis [12] and the Tau protein hypothesis [12]. It believes that the over-phosphorylation of $A \beta$-amyloid and Tau protein is hyperphosphorylated may be related to neuronal degenerative diseases, but not the main factor leading to degeneration of neurons (Figs. 1 and 2). Endogenous neurotransmitter regulation theory is a subversive theory, so the publication of the 


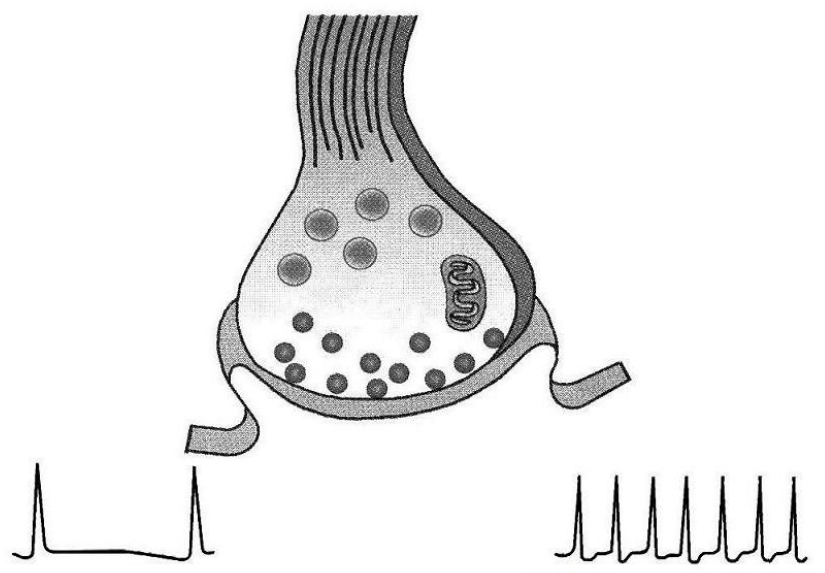

Low-frequency stimulation

High-frequency stimulation

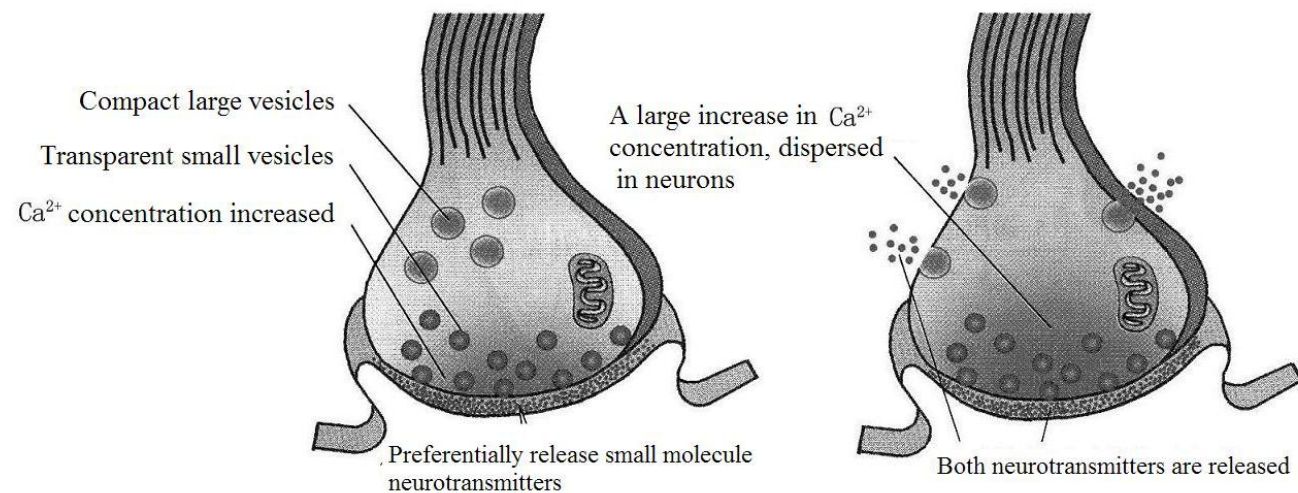

as the picture shows, Neurons in low-frequency stimulation, the Că channel on the presynaptic membrane is open, Ca concentration locally increased, peferentially release small-molecule neurotransmitters near the Ca channel; In the high-frequency stimulation, In the high-frequency stimulation, a large number of $\mathrm{Ca}$ into the neurons,Large and small molecule transmitter are released.

(Change fromPurves D, Williams SM. Neuroscience. 2nd ed.Sunderland:Sinauer Associates Inc, 2001.)

Fig. 1 Different neurotransmitters release probability is different.

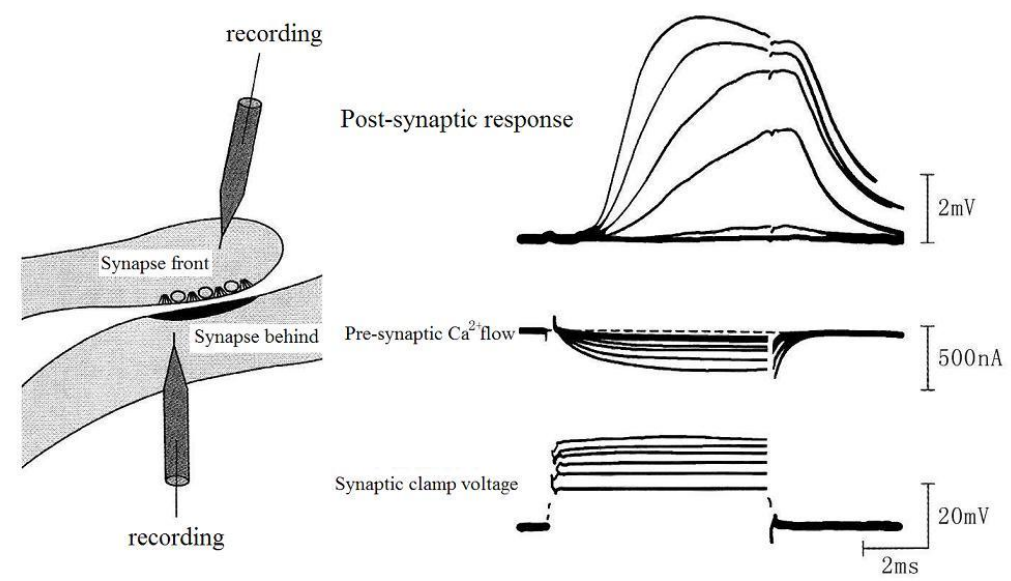

The electrodes recorded the presynaptic and postsynaptic fibers of squid synapse respectively, TTX and TEA were added to the external fluid to block the voltage-gated $\mathrm{Na}^{+}$and $\mathrm{K}^{+}$channels, respectively. Under the premise that presynaptic cells can not produce action potentials, Given a progressive depolarization of presynaptic progression, in addition to recording a gradual corresponding increase in presynaptic $\mathrm{Ca}^{2+}$ flow, a correspondingly increased postsynaptic response can also be recorded. That neurotransmitter release is not the result of the opening of $\mathrm{Na}^{+}$or $\mathrm{K}^{+}$ channels, but closely related to Cá influx.

(cited from Kandel ER, Schwartz JH, Jessell TM, Principles of Neuroscience. 4th ed. New York:McGraw-Hill, 2000.)

Fig. 2 Neurotransmitter release is the result of presynaptic terminal $\mathrm{Ca}^{2+}$ influx. 
paper is extremely difficult, but products based on this theory, such as PD therapy instrument and $\mathrm{AD}$ therapeutic instrument, have been highly recognized and concerned by peer scientists at home and abroad, making a large impact.

\subsection{Finding Points}

(1) We have found that voltage-gated calcium channels are the best targets for physical activation of neurons, proposed the regulation theory of endogenous neurotransmitters, and pointed out the principles and methods and purpose, for the treatment of neurodegenerative diseases such as PD and AD. It has clarified the reasons why medicinal chemistry can not fundamentally cure such diseases and the mechanism by which physical means can effectively treat such diseases.

(2) The spatial and temporal distribution of active treatment of encephalopathy caused by transcranial magneto-stimulation at a specific part of the head was found. And the corresponding relationship between the frequency, intensity, and time of transcranial magnetic stimulation to achieve regulation of endogenous neurotransmitters was established. It invented the endogenous neurotransmitter regulation technology and method, developed the transcranial magnetoelectric PD, AD therapeutic apparatus and realized industrial application.

\subsection{The Scientific Value of the Discovery Point}

(1) Endogenous neurotransmitter regulation theory supports the vesicle hypothesis and the cholinergic hypothesis, questioned the amyloid hypothesis and the Tau protein hypothesis, and pointed out the research directions of $\mathrm{PD}, \mathrm{AD}$ and other neurological degenerative disease rehabilitation treatment; (2) It first applied transcranial magnetic stimulation technology to develop $\mathrm{PD}, \mathrm{AD}$ therapeutic apparatus, made a breakthrough on the limitations that $\mathrm{PD}, \mathrm{AD}$ treatment mainly depends on drugs and surgery, and opened up new ways of physical means to treat neuronal degenerative diseases; (3) Changing no any means can prevent or delay the development of PD and $\mathrm{AD}$, reversing the over-reliance on the concept of drugs and surgery and the prejudice against physical means for the treatment of encephalopathy in the past; (4) Promoting the technological advancement of the brain health industry in the field of brain science has made our country lead the world by use of physical means to treat neurodegenerative diseases.

\section{Endogenous Neurotransmitter Regulation Method}

Endogenous neurotransmitters correspond to exogenous neurotransmitters. The balance between brain excitability and inhibitory neurotransmitter activity maintains the normal function of the brain, the neurotransmitter releasing into the synaptic cleft obeys the constant hypothesis of internal environment [13-15]. Transcranial magneto-stimulation technology can activate neurons and induce calcium influx to trigger the release of neurotransmitters from the axon terminal synaptic vesicles of the neurons, ensuring the activation of the synaptic cleft and inhibiting the balance of neurotransmitters. Long-term use of this treatment can restore the ability of neurons to self-generate and release neurotransmitters. Therefore, "transcranial magneto-stimulation technology" can also be understood as "endogenous neurotransmitter regulation technology or method".

\subsection{Propose Technical Ideas}

Only for PD and ad, which are the representative diseases of neuron degeneration.

According to the correspondence of PD, AD, neurotransmitters dopamine and acetylcholine, using transcranial magnetic stimulation technology and physical means of voltage-gated $\mathrm{Ca}^{2+}$ channels activate dopaminergic neurons and cholinergic neurons. Such as $\mathrm{PD} \rightarrow$ striatum $\rightarrow$ dopaminergic neurons $\rightarrow$ dopamine, $\mathrm{AD} \rightarrow$ (striatum, cerebral cortex, hippocampus $) \rightarrow$ cholinergic $\quad$ neurons $\rightarrow$ acetylcholine. 
Because, dopaminergic neurons are mostly concentrated on the striatum, cholinergic neurons are mostly concentrated on the striatum, cerebral cortex, hippocampus $[6,16]$.

2.1.1 Induction of $\mathrm{Ca}^{2+}$ Influx Triggers Release of Neurotransmitters in Neurons

Gating of $\mathrm{Ca}^{2+}$ channels on the cell membrane is "voltage", neuron excitement only responds to electrical signals [17, 18]. In different encephalopathies corresponding to different types of neurotransmitters in the main concentration area, find the best target area - cerebral cortex function area, the best target - the nucleus, the best target - the cell membrane calcium channel. Transcranial electrical stimulation of the whole brain at the same time, transcranial magnet also strongly stimulates the functional areas of different encephalopathies, thus strengthening the transcranial effect. Human brain in the cerebrospinal fluid, in theory, if the cerebrospinal fluid is energized, the current will be evenly distributed in the brain, even though the skull is an insulator, as long as current is introduced into the brain through the ear, a whole brain stimulus is formed. Skull is of high impedance, transcranial magnetic signals can pass through the skull and have a weak induction current in the brain, transcranial magnetic and transcranial complement each other, they activate neurons, and they induce calcium influx, which triggers the release of neurotransmitters from the terminal synaptic vesicles of neurons. Long-term use of it can restore the production of neurons self-generation, and release neurotransmitter function.

In addition, transcranial magnetoelectric stimulation can change the concentration of extracellular fluid cations.

2.1.2 To Ensure the Excitability of Synaptic Cleft and Inhibition of Neurotransmitter Balance

The balance between excitatory and inhibitory neurotransmitter activity maintains the normal function of the brain. The neurotransmitter releasing into the synaptic cleft obeys the constant hypothesis of internal environment, constant within the environment is not static, is based on a certain form of rhythm activity. Transcranial magnetoelectric stimulation plays a role of excitement and inhibition of both neurons, which is two-way, in theory it stimulates all the neurons, but when the site it plays a role, frequency, intensity are different, the release of neurotransmitters is also different, for example, macrophages neurons are far and late to release from the cellular $\mathrm{Ca}^{2+}$ channel. When a neuron is in an inhibited state, it can be activated to excite it, when a neuron is excited, it can be effectively suppressed.

Regeneration of endogenous neurotransmitters, including vesicle packing, transport and anchoring, requires both process and time. Calcium influx starts at the peak of action potential, and ends after the pre-synaptic membrane is fully repolarized. It makes the local area of calcium concentration from about 100 $\mathrm{nM}$ resting state rapidly increase to greater than $10-100 \mu \mathrm{M}$. The increase in calcium ion showed to be transient, the duration is about 400-500 $\mu \mathrm{s}$, time course corresponds to calcium current [19-21], synaptic vesicle fusion of molecular components requiring 1 to 5 calcium ions can trigger vesicle fusion or release. Synaptic neurotransmitter release at least is divided into synchronous rapid release and asynchronous slow release in two ways. The delay of synchronous release is very short $(50-500 \mu \mathrm{s})$, and asynchronous slow release can last $1 \mathrm{~s}$ or more [22, 23]. EEG biological rhythm synthesis frequency is of 8-13 Hz, catecholamine neurotransmitters by enzymatic inactivation, vesicle recycling process takes time: if stimulating by the frequency of $10 \mathrm{~Hz}$, usually it takes tens of seconds to a few minutes [24].

There was no linear relationship between vesicle fusion rate and free calcium concentration; making synaptic vesicle fusion is extremely sensitive to changes in calcium concentration and is limited to a very narrow range of calcium concentration and a short period of time [25]. Neuronal membrane depolarization leads to channel activation, but when 
activated to a certain extent, the channel is inactivated. It enters the state of no conductance, in the inactivated state, the voltage-gated channels are not activated anymore, even though the stimulus is still present or intense [6]. Therefore, the whole brain stimulation, may make the synaptic cleft excitement and inhibition of neurotransmitters appear instant imbalance, but will not lead to normal neurons uncontrolled release of neurotransmitters for a long time, for example, patients with PD may experience more transient jitter after using transcranial magnetoelectric stimulation, but calmness will soon resume.

In fact the body internal activity of the nerve is fluctuating, which is best illustrated by the feedback of regulation in the hypothalamus-pituitary-gland axis [26, 27]. Therefore, "transcranial magnetoelectric stimulation technology" can also be understood as "endogenous neurotransmitter regulation technology" or "neural regulation technology" (shown in Fig. 1).

\subsection{On the Physical Means}

Magnetic stimulation [28], magnetic twitch therapy, using time-varying magnetic field in vivo induced electric field and produced induced current. Electrical stimulation, that is, electroconvulsive therapy, begins in the early nineties of last century. Because of inadequate treatment mechanisms and safety issues, magnetic twitch and electroconvulsive therapy are limited to the treatment of severe mental illness and chronic refractory pain, and are only allowed to be used by professionals in the clinical setting.

After the technology search and literature search, internationally, the first application of transcranial electrical stimulation technology for the treatment of stroke sequelae, cerebrovascular dementia and brain atrophy and other neuronal ischemic encephalopathy, is by the first author of this article who invented transcranial computer functional rehabilitation apparatus, beginning in 1995. In 1994, his loved one suffered a brain stroke and then he invented a transcranial computer functional rehabilitation instrument. In 1995, the product obtained the national medical device product registration certificate. In 1996, accessing to national patents, at the same year he founded Harbin Aobo Medical Devices Co., Ltd., and transcranial computer functional rehabilitation instrument achieved the industrial application. In 1998, the author and clinical experts co-published the first application of transcranial electrical stimulation as the core technology for the treatment of encephalopathy clinical observation of the paper "Effects of Aobo brain function rehabilitation instrument on cerebral circulation and brain function" [29].

Transcranial electricity brain function rehabilitation treatment instrument was developed successfully and laid the technical foundation for the transcranial magnetic stimulation technology research and application. It also provides academic directions for domestic and foreign scholars to study transcranial electrical stimulation technology. In the following 20 years, the first author conducted a long-term, in-depth and systematic exploration and practice from two aspects of the basic theoretical research and clinical practice of brain science. He proposed "the theory of brain cell activation", in 2011 and 2014, he invented Parkinson therapy instrument [30, 31], Alzheimer treatment instrument [30,32], depression (insomnia) treatment instrument $[33,34]$ and other transcranial magnetic computer series of rehabilitation treatment equipment, changed the past people's treatment of encephalopathy which mainly relied on the concept of drugs and surgery. It provides a new way of physical treatment for the treatment of degenerative diseases of neurons.

\subsection{Comparison of Similar Studies}

Magnetic stimulator at home and abroad is mainly round coil or 8-shaped coil-based. Stimulation output is high-pressure and low-frequency pulsed magnetic field, the actual output of 1-3 T (Tesla) or even higher. The main result of the project using transcranial magnetic E-shaped coil is a multi-turn magnetic field 
generator, multi-point low-frequency alternating magnetic field, the actual output does not exceed 50 $\mathrm{mT}$ (milli-teres), the target is the superficial cortex of the brain, the magnetic stimulation intensity reaching the target is $1-2 \mathrm{G}$ (Gauss) $(1 \mathrm{~T}=10,000 \mathrm{G})$. Domestic and international electricity twitch therapy, beginning in the early nineties of last century, is a high voltage current, usually more than $90 \mathrm{~V}$. The main results of this project using transcranial electrical stimulation are low voltage and weak current.

Monkey research shows that, as long as 1-2 G magnetic stimulus is reached in the cerebral cortex, the threshold of hippocampus and motor cortex will be increased. When the cell membrane potential is close to $-40 \mathrm{mV}$, the probability of opening $\mathrm{Ca}^{2+}$ channel began to significantly increase. Whether it is transcranial magnetic or transcranial electrical, neurons are the ultimate role of "electricity", the $\mathrm{Ca}^{2+}$ channel on the cell membrane gating way is also "electricity", therefore, neurons in the repressed state only respond to electrical signals.

The transcranial magnetic stimulation technology used in this project is a noninvasive technique. It is weak magnetic micro-current stimulation, and is the second national medical equipment. Its mechanism is clear, and it has no side effects of safety, can be used by encephalopathy patients in hospitals and families.

The main outcome of this project, Alzheimer therapeutic apparatus, is original in the world, no similar products; another major achievement of this project, Parkinson treatment instrument, is similar to and comparable to, brain pacemakers in the United States.

American brain pacemaker, equivalent to human "brain pacemaker", is an invasive deep brain stimulation, which can significantly improve and control tremor, rigidity, bradykinesia and other symptoms. Its damage to the brain structure is reversible, not affecting taking other new treatment methods in future. The application of the theory of brain cell activation can explain its treatment mechanism. The main achievement of this project, Parkinson treatment instrument, is a multi-point, multi-turn, multi-channel transcranial magnetic neuromodulation system, which is equivalent to people's "brain pacemaker in vitro". It is non-invasive deep brain stimulation, suitable for mild to moderate Parkinson's disease, and can significantly improve the resulting tremor, stiffness, bradykinesia and other symptoms. The theoretical basis is the theory of brain cell activation divided into two types of medical and household, which can enter the hospital into the family.

\subsection{Transcranial Magnetoelectric Encephalopathy}

\section{Treatment Instrument}

Parkinson therapy instrument, Alzheimer treatment instrument and other rehabilitation equipments of transcranial magnetic computer disease series, made the first application of transcranial magnetic stimulation technology to treat $\mathrm{PD}, \mathrm{AD}$, made a breakthrough on the limitations of $\mathrm{PD}, \mathrm{AD}$ treatment mainly depending on drugs and surgery, opened up a new approach to physical treatment of neuronal degenerative disease.

Transcranial magnetoelectric encephalopathy treatment instrument is a multi-bit, multi-turn, multi-channel transcranial magnetic nerve stimulation system, which includes head-mounted field effect caps and EEG generators. Of which: EEG analog generator, is a kind of brain cells that can activate the instrument, the output of a special electrical signal to simulate the brain wave of healthy people, frequency and intensity, can be benign and weak electrical stimulation of neurons; Field effect caps, containing magnetic devices, for different types of brain diseases choose the best target for human head.

Technical solutions: transcranial magnetoelectric is generated by three-dimensional superimposed alternating magnetic field and pulse current in the brain co-acting on deep brain delivery of energy neurons, transcranial magnetic brain in a particular 
part of the formation of three-dimensional superimposed alternating magnetic field induced weak induction current, can direct the activation of cortical neurons; transcranial electrical pathway in the basal ganglia neurons through the nucleus, the enhanced transcranial magnetic effect, transcranial magnetic and transcranial complement each other, taking into account the fact that neurons transmit neurons and skull high impedance distribution (Figs. 2 and 3 ).

\section{The Application of Neural Regulation Theory Method}

From the hypothesis of encephalopathy treatment to the creation of "The Theory of Brain Cell Activation"; from the transcranial electrical stimulation from the invention of technology to formation of a transcranial magnetic core patented technology; from the development of the first brain rehabilitation therapy apparatus to development of a transcranial magnetic computer treatment of products, from practice to theory, to practice again, after more than 20 years, involved in the treatment of major diseases of neuronal degenerative diseases, ischemic encephalopathy, mental illness, a total of 4 countries registered medical device products, which are independent intellectual property rights, and all realized the industrialization.

\subsection{Transcranial Magnetoelectric Encephalopathy} Treatment Instrument (AOBO Parkinson Treatment Instrument)

The first registration date is January 31, 2011; medical device product registration number: HEI SHI YAO JIAN (prospective) word 2011 No. 226001; scope: mild to moderate Parkinson's disease, it can significantly improve the resulting tremor, stiffness, bradykinesia and other symptoms [35].

Parkinson treatment instrument, selects voltage-gated $\mathrm{Ca}^{2+}$ channel as the best target of transcranial magnetoelectric effect. The purpose is to
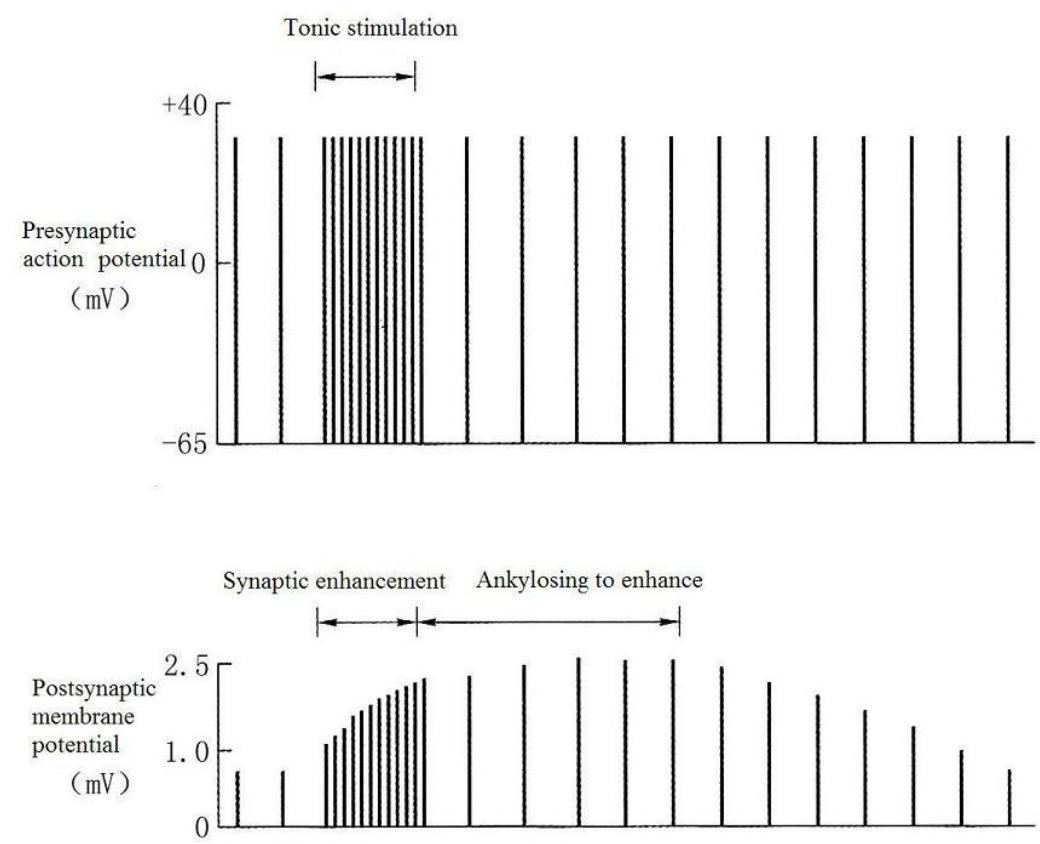

As comparison, First give several presynaptic neurons $1 \mathrm{~Hz}$ stimulation, induced presynaptic action potential, postsynaptic neurons can record postsynaptic potentials of about $1 \mathrm{mV}$. A series of $5 \mathrm{~Hz}$ tonic stimulation of presynaptic neurons within the ensuing few seconds, at the same time, a gradual increase in postsynaptic potential was recorded. When the presynaptic neuron stimulation frequency returned to $1 \mathrm{~Hz}$, the post-synaptic response is still maintained for some time enhanced response, that is, after strengthening, and then gradually reduced.

(Change from Kandel ER, Schwartz JH, Jessell TM, Principles of Neuroscience. 4th ed. New York:McGraw-Hill, 2000.)

Fig. 3 Synaptic enhancement and post-ankylosing enhancement produced by tonic stimulation. 
induce $\mathrm{Ca}^{2+}$ influx trigger neuronal axon terminal synaptic vesicles release neurotransmitter, activate dopaminergic neurons [6, 9], break through the limitations on international treatment of PD mainly relying on drugs and surgery, equivalent to human brain pacemaker, and it is another new way to treat PD.

Completed sci-tech novelty retrieval and results identification, to fill gaps at home and abroad, transcranial magnetoelectricity technologies in the field of physical therapy of PD live in the international advanced level, for the first of the world. Parkinson treatment instrument, has been listed as key scientific and technological projects in Heilongjiang Province, supporting projects that the Ministry of Science and Technology Innovation Fund focuses on, the first key areas (sets) of products identified by the Committee of Industry and Information Technology of Heilongjiang Province, won the first prize of Heilongjiang Province Pharmaceutical Industry Science and Technology Progress Award, China Industry-University-Research Cooperation Innovation Award, the first prize of Heilongjiang Province Science and Technology Invention Award, and is listed as Heilongjiang Province "Twelve Five" projects key to promote industrialization and projects for structural adjustment of biological industry in Heilongjiang Province.

\subsection{Transcranial Magnetoelectric Encephalopathy} Treatment Instrument (AOBO Alzheimer Treatment Instrument)

The first registration date: April 17, 2014; medical device product registration number: HEI SHI YAO JIAN XIE (prospective) word 2014 No. 2260036; scope: mild to moderate Alzheimer's disease, vascular dementia, improving the patient's mental state, cognitive behavior and ability to better take care of their own daily life [32].

Alzheimer treatment instrument, selects voltage-gated $\mathrm{Ca}^{2+}$ channel as the best target of transcranial magnetoelectric effect. The purpose is to induce $\mathrm{Ca}^{2+}$ influx trigger neuronal axon terminal synaptic vesicles release neurotransmitter, activate cholinergic neurons [9], open up new ways of treating $\mathrm{AD}$ by physical means.

Completed sci-tech novelty retrieval and results identification, to fill gaps at home and abroad, transcranial magnetoelectricity technology in the field of physical therapy of $\mathrm{AD}$ lives in the international advanced level, for the world's first. Alzheimer treatment instrument, is the key scientific and technological projects of Heilongjiang Province and Harbin City, has won the Harbin Science and Technology Progress Award, won the first prize of Heilongjiang Province Pharmaceutical Industry Science and Technology Progress Award, and is listed as Heilongjiang Province "Twelve Five" projects key to promote industrialization and projects for structural adjustment of biological industry in Heilongjiang Province.

\subsection{Transcranial Magnetoelectric Depression (Insomnia) Treatment Instrument}

The first registration date is January 31, 2011; medical device product registration number: HEI SHI YAO JIAN (prospective) word 2011 No. 226002; scope: mild to moderate depression, it can significantly improve depression, sleep disorders, anxiety and other major symptoms [34].

Transcranial magnetoelectric depression (insomnia) treatment instrument, selects voltage-gated $\mathrm{Ca}^{2+}$ channel as the best target of transcranial magnetoelectric effect, the aim to activate serotonergic neurons [9].

Completed sci-tech novelty retrieval and identified provincial new product by Committee of Industry and Information Technology of Heilongjiang Province, to fill the gaps at home and abroad, for the first around the world, the core technology is in the international advanced level in the field of depression treatment applications. Transcranial magnetoelectric depression (insomnia) treatment instrument, has been included in 
the 2011 national key development plan of new products, the 2012 Torch Program project, won the first prize of the eighth Heilongjiang Province outstanding new products, the first prize of Heilongjiang Province Pharmaceutical Industry Science and Technology Progress Award, the first prize of Heilongjiang Province Science and Technology Award (invention class).

\subsection{Transcranial Electricity Brain Function Rehabilitation Treatment Instrument}

The first registration date is on December 4, 1995; medical device product registration number: HEI YI XIE ZHUN ZI (95) No. 227014; scope: stroke sequelae, cerebrovascular dementia and brain atrophy $[29,36]$.

Transcranial electricity brain function rehabilitation treatment instrument, selects voltage-gated $\mathrm{Ca}^{2+}$ channel as the best target of transcranial effect, and the goal is to save the dying neuron and activate the neuron in the repressed state $[9,10]$.

Stroke, whether it is ischemic cerebrovascular disease or hemorrhagic cerebrovascular disease, once into the sequelae, belongs to ischemic encephalopathy, such as brain atrophy or cerebrovascular dementia, having the same treatment mechanism with $\mathrm{AD}$, and the method is similar: activation of energy-producing neurons is the key to treatment. Transcranial electricity brain function rehabilitation treatment instrument, has activated key areas of neuronal groups taking into account the whole brain stimulation, for stroke sequelae, cerebrovascular dementia and brain atrophy encephalopathy, and opened up a new way for the treatment of ischemic encephalopathy.

In 1995 the Heilongjiang Province Food and Drug Administration organized the provincial scientific and technological achievements appraisal. Identification conclusion: the product is to fill the gaps in the country, in the advanced level of domestic and international similar products. Transcranial electricity brain function rehabilitation treatment instrument, has successively been listed as a major scientific and technological project in Heilongjiang Province and a major science and technology industrialization project, National Torch Plan Project, key support projects of National Science and Technology Enterprise Innovation Fund, won China Invention Exhibition patent gold medal, International Invention Expo Repin Repin Award in Paris, France, the first prize of scientific and technological benefit in Heilongjiang Province, the first prize of Heilongjiang Province Science and Technology Award (invention class).

\section{Conclusion}

Life sciences, especially neuroscience, have been studied to the very micro level. Many biochemical results are obtained by physical means, and the theory of brain cell activation is also based on the clinical practice of transcranial magnetic stimulation technology and the existing biochemical results that derived a conclusion. Regrettably, when treating various diseases, we often emphasize one and underestimate the other, and few mention physical means, or just locate it on adjuvant therapy, ignoring the complementary relationship between physical and chemical means.

The theory of brain cell activation suitable for encephalopathy is not limited to encephalopathy, based on this, you can create "Discipline of Physical Diseases". According to the gating of $\mathrm{Ca}^{2+}$ channels, the disease can be divided into physical diseases, chemical diseases and physical and chemical diseases. When the cell is in an inhibited state, it only responds to the electrical signal; when the cell is in an excited state, it is possible for the chemical to flatten it from the excited state to the normal state, which can be done through physical means. Namely, the diseases that can be solved by drugs are also possible through physical means. The problems that drugs can not solve can be solved by physical means.

Divided simply from the cell inhibition and excitement, physical means are with two-way 
adjustment. Anyone who has a neurodegenerative disorder that is selectively degenerative, which belongs to the physical gated $\mathrm{Ca}^{2+}$ channel disease, can try to be treated by physical means, such as schizophrenia, bipolar disorder, myasthenia gravis, Huntington's disease, epilepsy, pediatric cerebral palsy, autism, mental retardation, addiction, CO poisoning, pain, vegetative disease, coronary heart disease, diabetes, more than 20 kinds of major diseases.

In fact, physical means, especially transcranial magnetoelectric stimulation technology, has aroused the widespread concern of the world's scientists. In December 2016, a number of internationally renowned brain scientists jointly issued a long article on the well-known academic journal Neuron, giving high attention and affirmation to the emerging physical means of transcranial stimulation [37]. In June 2017, more than 10 scientists from a number of authoritative academic institutions such as Massachusetts Institute of Technology, Harvard Medical School, and Royal London Hospital conducted a basic experimental study on noninvasive transcranial low-frequency electrical stimulation. And in the Cell magazine published an article: "Transcranial low-frequency electrical stimulation technology is mature and reliable. It is recommended that you do not have to do the brain biochemical test for the brain, call for such an approach to be applied as soon as possible in the treatment of major human brain diseases, such as the treatment of PD, AD, stroke sequelae and the study of the human brain" [38].

In recent years, the reward objects of international authoritative scientific and technological awards organizations have also begun to favor physical therapy, at the same time also attracted the attention of strategic investors. In 2014, inventors of brain pacemakers Mahlon Delong and Alim Louis Benabid won the Lasker Award, the highest award in medical science in the United States. In 2013 and 2015, it won the Scientific Breakthrough Award again and again. GlaxoSmithKline invested heavily in 2013 to support research similar to the main results of this topic, in 2016, Neuronix Company of the United Kingdom combined transcranial magnetic stimulation and cognitive training, "Targeted Encephalopathy Area for Alzheimer's Disease", the treatment of mild to moderate Alzheimer's disease (AD) has a positive effect, and approvals from the U.S. Food and Drug Administration (FDA) are being sought [39, 40]. Bill Gates also said in 2017 that it will invest heavily in research on the physical therapy of $\mathrm{PD}$ and $\mathrm{AD}$ other than drug development.

Method and application of endogenous neurotransmitter regulation theory, once again reminded the industry professionals in the chemical synthesis at the same time to not ignore the physical means, the combination of physical means or the combination of physical and chemical means which may be one of the most important research directions in the future for the total overcoming of neurodegenerative diseases in humans. In the face of 40 million $\mathrm{PD}, \mathrm{AD}$ patients and their families who are in pain, the face and interests of any organization must be unconditionally put down.

Last, take this opportunity to publish the paper, I want to revise a paper that has been published in your journal--Interpretation of Action Potential Generation Mechanism in Cells by Potassium Channel "Origami Windmill" Model. The lowest measured value of membrane potential in Figure B is $-60 \mathrm{mV}$ instead of $-65 \mathrm{mv}$ as described in the paper. Therefore, "-65mv" in the paper should be revised to "-60mV". In the corresponding paper, "the falling phase is more than the rising phase by $25 \mathrm{mV}$ " revised to "the falling phase is more than the rising phase by $20 \mathrm{mV}$ ". Necessity of revision: Because the lowest point of measured membrane potential is $-60 \mathrm{mV}$, and the highest point is $40 \mathrm{mv}$. Their absolute value ratio is exactly $3: 2$. This ratio is very intuitive and can explain some problems. 


\section{References}

[1] Zhang, Z. X., Roman, G. C., Hong, Z., et al. 2005. "Parkinson's Disease in China: Prevalence in Beijing, Xian, and Shanghai." Lancet 365 (9459): 595-7.

[2] Chen, W., Xu, Z. M., Wang, G., et al. 2012. "Non-motor Symptoms of Parkinson's Disease in China: A Review of the Literature." Parkinson's Relat Disord 18 (5): 446-52.

[3] Gui, Y. X., Wan, Y., Xiao, Q., et al. 2011. "Verification of Expressions of Kir2 as Potential Peripheral Biomarkers in Lymphocytes from Patients with Parkinson's Disease." Neurosci Lett 505 (2): 104-8.

[4] Chen, S. D., Wang, G., Liu, J., et al. 2012. "The Basic and Clinical Research Progress of the Pathogenesis and Diagnosis and Treatment of Parkinson's Disease." Journal of Shanghai Jiao Tong University (Medical Edition) 32 (9): 1221-6.

[5] Wang, X. J., Zhang, Y., and Chen, S. D. 2010. "Development in the Continue Decade of Pathogenesis of Parkinson's Disease and Treatment Studies." Chin J Contemp Neurol Neurosurg 10 (1): 36-42.

[6] Sun, Z. D. 2010. Conquest of Parkinson. Harbin: Heilongjiang science and Technology Press.

[7] Cheng, J. Y., Liang, Q. C., Wu, Y., et al. 2017. "Advances in the Pathogenesis and Drug Treatment of Alzheimer's Disease." Progress in Modern Biomedicine 17 (10): 1981-5.

[8] Ge, R. 2014. "Developments Research on Alzheimer's Disease." Chinese and Foreign Medical Research 12 (9): 155-7.

[9] Sun, Z. D. 2016. Theory of Brain Cell Activation. Harbin: Heilongjiang Science and Technology Press.

[10] Sun, Z. D. 2003. Activate the Sleeping Brain. Harbin. Heilongjiang People's Publishing House.

[11] Han, J. S. 2009. Neuro Science, 3rd ed., Beijing: Peking University Medical Press.

[12] Maruyama, M., Shimada, H., Suhara, T., et al. 2013. "Imaging of Tau Pathology in a Tauopathy Mouse Model and in Alzheimer Patients Compared to Normal Controls." Neuron 79 (6): 1094-108.

[13] Ahima, R. S. 2005. "Central Actions of Adipocyte Hormone.” Trends Endocrinol Metab 16: 307-13.

[14] Bichet, D. G. 1997. "Posterior Pituitary Hormones." In Endocrinology: Basic and Clinical Principle. New York: Human Press, 223-45.

[15] Gekakis, N., Staknis, D., Nguyen, H. B., et al. 1998. "Role of the CLOOK Protein in the Mammalian Circadian Mechanism." Science 280: 1564-8.

[16] Yang, X. L. 2014. Neurobiology: From Neurons to the Brain, 5th ed., Beijing: Science Press.

[17] Chen, Y. Z. 2014. Synapse. Shanghai: Shanghai Science and Technology Press.
[18] Bear, M. F. 2004. Neuroscience: Explore the Brain. Beijing: Higher Education Press.

[19] Bear, M. F., Connors, B. W., Paradiso, M. A. 2001. Neuroscience: Exploring the Brain. Philadelphia: Lippincott Williams, Wilkins Inc., 99-161.

[20] Burgoyne, R. D., and Morgan, A. 2003. "Secretory Granule Exocytosis." Physiol Rev 83: 581-632.

[21] Jahn, R., Lang, T., and Sudhof, T. C. 2003. "Membrane Fusion." Cell 112: 519-33.

[22] Kandel, E. R., and Siegelbaum, S. A. 2000. Principles of Neural Science. New York: McGrqaw-Hill, 175-86, 253-79.

[23] Bhalla, A., Chicka, M. C., Tucker, W. C., et al. 2006. " $\mathrm{Ca}^{2+}$-Synaptotagmin Directly Regulates t-SNARE Function during Reconstituted Membrane Fusion." Nat Struct Mol Biol 13 (4): 323-30.

[24] Heuser, J. E., and Reese, T. S. 1973. "Evidence for Recycling of Synaptic Vesicles Membrane during Transmitter Release at the Frog Neuromuscular Junction." Cell Biol. 57: 315-44.

[25] Rettig, J., and Neher, E. 2002. "Emerging Roles of Presynaptic Proteins in $\mathrm{Ca}^{2+}$-Triggered Exocytosis." Science 298: 781-5.

[26] Taheri, S., Zeitzer, J. M., and Mignot, E. 2002. "The Role of Hypocretins (Orexins) in Sleep Regulation and Narcolepsy.” Annu Rev Neurosci 25: 283-313.

[27] Zhu, Y., Bond, J., and Thomas, P. 2003. "Identification, Classification, and Other Vertebrates Homologous to a Fish Membrane Progestin Receptor." Proc Natl Acad Sci USA 100: 2237-42.

[28] Adelman, G. 1994. Encyclopedia of Neuroscience. Shanghai: Shanghai Science and Technology Press.

[29] Jiao, M. D., and Sun, Z. D. 1998. "Effects of Aobo Brain Function Rehabilitation Instrument on Cerebral Circulation and Brain Function." Medicine Healthcare Apparatus 3: 251-2.

[30] Tian, N. N. 2009. "Application of Aobo Brain Function Rehabilitation Instrument in Post-Troke Hemiplegia Patients." Chinese Journal of Medical Device 9: 68.

[31] Sun, Z. D. 2012. Transcranial Magnetoelectric Encephalopathy Therapeutic Instrument, China ZL200910071875.X, 2012-08-22.

[32] 2011. "Successful Development of the First Therapeutic Instrument for Parkinson's Disease in China." Journal of Minimally Invasive Medicine 6 (4): 314.

[33] 2014. "Successful Development of the First Therapeutic Instrument for Alzheimer Disease in the World." Science-Technology \& Publication 6: 143.

[34] Sun, Z. D. 2011. Transcranial Magnetoelectric Depression Therapeutic Instrument, China, ZL200910071876.4., 2011-08-24.

[35] 2011. "Harbin Successfully Develops the First 
Therapeutic Instrument for Depression in the World." Science-Technology \& Publication 6: 127.

[36] Xing, X. L., and Tang, Q. 2011. "Clinical Research on Influences of Transcranial Magnetoelectric Stimulation on Parkinson's Disease." The Assembly of Conference Papers of the 11th National Rehabilitation Academic Conference of Exercise Therapy Branch of Chinese Association of Rehabilitation Medicine.

[37] Poo, M. M., Du, J. L., Ip, N., et al. 2016. "China Brain Project: Basic Neuroscience, Brain Diseases, and Brain-Inspired Computing.” Neuron 92 (3): 591.
[38] Grossman, N., Bono, D., Dedic, N., et al. 2017. "Noninvasive Deep Brain Stimulation via Temporally Interfering Electric Fields." Cell 169 (6): 1029.

[39] Gonsalvez, I., Baror, R., Fried, P., et al. 2017. "Therapeutic Noninvasive Brain Stimulation in Alzheimer's Disease." Current Alzheimer Research 14: 1-15.

[40] Rabey, J. M., and Dobronevsky, E. 2016. "Repetitive Transcranial Magnetic Stimulation (rTMS) Combined with Cognitive Training Is a Safe and Effective Modality for the Treatment of Alzheimer's Disease: Clinical Experience." J Neural Transm 123: 1449-55. 